\title{
Methods of Automatic Control of the Topological State of the Equipment of Electrical Substations
}

\author{
Ilya Abramovich Golovinskii ${ }^{1}$ \\ ${ }^{1}$ OJSC "Zelenograd innovation and technology centre" (ZITC), Moscow, Russia \\ Correspondence: Ilya Abramovich Golovinskii, OJSC "Zelenograd innovation and technology centre" (ZITC), \\ 124498, Moscow, Russia. E-mail: igolovinskij@gmail.com
}

Received: November 17, 2014

Accepted: November 27, 2014

Online Published: December 10, 2014

doi:10.5539/mas.v9n4p53

URL: http://dx.doi.org/10.5539/mas.v9n4p53

\begin{abstract}
Topological states of substations' equipment are determined by the states of switching devices. The considered classes of topological states are the states of: individual devices, groups of electrical devices, and substations as a whole. Along with independent significance of these characteristics their role as logical locks when switching is considered. Topological characteristics are calculated by analyzing the topology of electrical circuits and substations. For this, the mathematical formalism has been developed, which is based on the special algebra of graphs. Formulas are given to calculate the topological states of the equipment of substation.
\end{abstract}

Keywords: electrical network, substation control, operational state of electrical installation, topological state of substation equipment, analysis of contact circuits, topological processor, logical locking of switchings

\section{Introduction}

\subsection{Task Description}

In the technology of operative-dispatch control of electrical networks one of the key tasks is to monitor the operational status of substations. It is defined by the operability of substation equipment, the position of switching devices, the setting of controlled devices. The aspect of the operational state of the power substation, which is determined only by the position of the switches will be called as the topological state of the substation. Its characteristics are such properties of the substation mode as, for example, the separation of the substation into the independently operating parts, the presence of transit through the substation, the presence of voltage on the bus sections, the presence of the load on the devices, the connection of power lines with the bus sections, etc. Further article there will be considered other characteristics of topological states of substation equipment. All of them will be called as the topological characteristics of the substation.

During planning any switching operations, both for normal or emergency conditions, we must know the characteristics of such substation topological state, which should result from the implementation of the planned switching operation. To do this, you must "play" the planned switching operation on the simulation model of the substation. If as a result of the planned switching operation the topological state occurs, which is unacceptable on the technical regulations criteria, the planned switching operation should be canceled or its implementation should be blocked. In the case of switchings, produced by automatic devices, the analysis of topological characteristics of the substation must be performed on actually occurring state of substation equipment.

Identification of the current substation equipment topological state is often a complex logical problem. Minimizing the time consumption on its decision and the probability of errors in logical analysis is achieved by automated solutions. The content of the article is constituted by mathematical methods that enable this automation.

The necessary condition for the implementation of the control of the topological state of the substation is the availability of complete information about the current status of its switches. The matter of getting these data is not considered in the article. It is assumed that all these data have been obtained by various methods (Lubarsky, 2003; Gikinskaya, \& Lyubarskii, 2003; Gikinskaya, et al., 2007). From the formulas, given in the article, it is clear what the source data are required to solve a particular problem. 


\subsection{Topological States of Substation Equipment}

Automatic control of the topological state of the substation is provided by the online program Switching States Monitor (SSM). It is started with a certain periodicity, as well as by events - by each single switching and by group of interconnected switchings, caused by the single or cascade triggering of protection and automation devices.

According to the input data the SSM recognizes, for what substation equipment it is necessary to update the estimation of the topological state. Such equipment may be a separate device (power line, power (auto)transformer, bus section, reactor, breaker et al.), group electrical installation, or substation as a whole.

We use the concept of the group electrical installation to describe the substation topological states, which are determined by the topology of the electrical connection of several power devices. In dispatching management of electrical networks a control is chiefly required for the following types of group electrical installations:

- switchgear: a set of bus sections in the substation, interconnected by the switches;

- a pair of parallel transformers: a pair of power (auto)transformers, which have identical technical characteristics and allow parallel operation by their interconnecting at all their voltage levels;

- a power line join with the switchgear;

- a group of lines for possible power transit through the substation: a set of power lines, which have the highest voltage class for this substation and are connected with each other within substation switchgear (i.e. through the circuits without transformers). These power lines can carry out a transit of electricity through the substation.

Characteristic of the topological state of the substation is made up of the characteristics of the topological state of its equipment. For individual power devices SSM should provide the recognition (identification) of the following topological states:

- on load;

- under voltage;

- off (disconnected);

- separated by visible break (isolated);

- isolated and grounded.

The state "on load" means that a load current flows through the device. The state "under voltage" occurs when a voltage is applied to the device. "Off (disconnected)" mode exists when the device is disconnected from the power sources. Device status "separated by visible break" (from the sources and consumers) means the open state of all its disconnectors, etc.

Table 1. Topological states of the group electrical installations

\begin{tabular}{|c|c|c|}
\hline $\begin{array}{l}\text { The type of the group electrical } \\
\text { installation }\end{array}$ & $\begin{array}{r}\text { The name } \\
\text { topological ch }\end{array}$ & Possible \\
\hline Switchgear & Connectivity & $\begin{array}{l}\text { - Joint operation } \\
\text { - Separate operation (listing the jointly } \\
\text { operating bus sections) }\end{array}$ \\
\hline A pair of parallel $t$ & ion parallelism & $\begin{array}{l}\text { - Parallel operation } \\
\text { - Joint nonparallel operation } \\
\text { - Separate operation }\end{array}$ \\
\hline $\begin{array}{l}\text { The join of the power line with } \\
\text { the switchgear }\end{array}$ & $\begin{array}{l}\text { The presence of the } \\
\text { electrical connection }\end{array}$ & $\begin{array}{l}\text { nnection is present (listing the joined } \\
\text { ons) } \\
\text { nection }\end{array}$ \\
\hline $\begin{array}{l}\text { A group of lines for possible } \\
\text { power transit through the } \\
\text { substation }\end{array}$ & The presence of the transit & $\begin{array}{l}\text { - The transit is present (listing all the power } \\
\text { lines' groups, which implement the transit) } \\
\text { - No transit }\end{array}$ \\
\hline
\end{tabular}

Possible topological states (topological modes) for group electrical installations are shown in Table 1. Let us explain topological modes for such group electrical installation, as a pair of parallel transformers. For a pair of two-pole transformers, the state "parallel operation" means a connection via closed chains within the switchgear 
at both the high-voltage (HV) and low-voltage (LV) levels. If there is a closed chain within the switchgear only at HV level and the LV side has no such chains, then this topological mode is called "joint nonparallel operation". Topological mode of pair of transformers with the absence of closed chains both at the HV side and LV side within the switchgear will be called "separate operation". The fourth case is the absence of closed chains within the switchgear at HV side in the presence of such a circuit at the LV side. The technology of operational control of electrical networks allows this mode only for a short time when performing the switching operations.

Respectively, for the three-pole transformers the "parallel operation" will mean the presence of closed chains between these transformers within the switchgear at the HV, LV and medium voltage (MV). The "separate operation" means the absence of such chains at all sides. All other long-time configurations allowed by the operational control technology are referred to the switching mode "joint nonparallel operation". The mode is allowed only for a short time, if at the HV side there is no connection via closed chains within the switchgear and there is a closed chain at the MV or LV side.

To control the topological state of the substation as a whole object we need to know, in addition to the topological characteristics of its individual devices and group installations, also the topological characteristics of the substation as a whole, which are irreducible to the topological characteristics of the parts of substation. An example of such features is the description of the substation separation into parts that are not interconnected via the closed chains. Other examples are the descriptions of the substation separation between the voltage levels and the transit violations at the highest voltage level.

\section{Methods}

A set of methods of detection of topological characteristics of substations will be called more briefly switching circuits' analysis. This set is based on a logical analysis of the states of the switches and the topology of their connections. The automation of the switching circuits' analysis consists in implementation of this logic in computer programs.

A mathematical method of the switching circuits' analysis, explained below, belongs to the topological trend in the analysis of contact circuits. In the works of the founders of mathematical theory of relay-contact circuits A. Nakashima, C.E. Shannon and V.I. Shestakov the formalism of the Boolean algebra was used, but the information about the topology of the contact circuit was not involved (Stanković, et al., n.d.). The first of the topological methods of the analysis of the contact circuits was the application of the algebra of Boolean matrices, which are the incidence matrices of the contact circuits' graphs. This method was developed in the Leningrad Electrotechnical Institute in the second half of the 1940s. (Note 1). The idea and the mathematical formalism were offered by A.G. Lunts (Lunts, 1950a; Lunts, 1950b; Lunts, 1952), the application to practical problems was developed by B.I. Aranovich (Aranovich, 1947; Aranovich, 1949). Another topological method of the switching circuits' analysis originated independently in the electric power industry. This was the method of semantic networks. This method was developed by Y.Y. Lubarskiy and his assistants since the 1970s. (Lubarskiy, 1978; Kupershmidt, et al., 1985).

To perform the switching circuits' analysis, we need a database of the control complex SCADA/EMS, which contains a description of the network topology. Naturally, the connections of the elements of the circuits should be represented as undirected graph. The most detailed and complete description of the electric circuits is provided by CIM (Common Information Model) standard (IEC 61970-301, n.d.; IEC 61968-11, n.d.; Uslar, et al., 2012). Less detailed, but sufficient description for the analysis of switching circuits, gives the stellar graph, or a graph of the stellar model (see Note 2). The use of stellar model is more convenient than that of CIM to describe the application of the offered methods of switching circuits' analysis. 


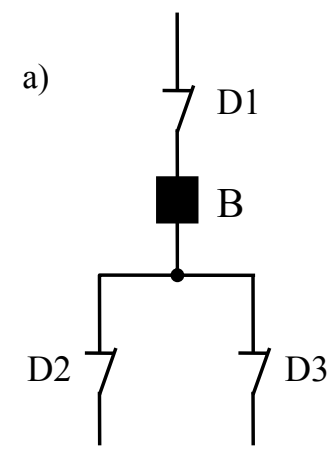

b)

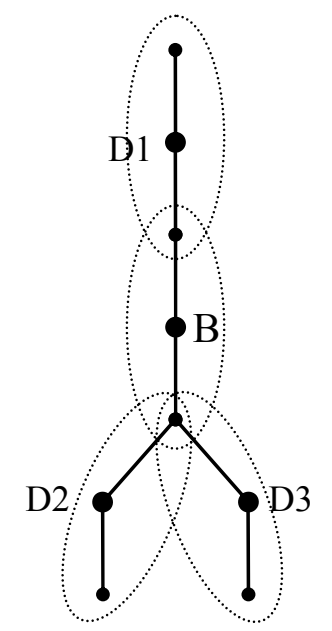

Figure 1. The breaker (B) and three adjacent disconnectors (D1, D2 и D3): $\mathrm{a}$ - the pictorial image, $\mathrm{b}$ - the corresponding fragment of the stellar graph

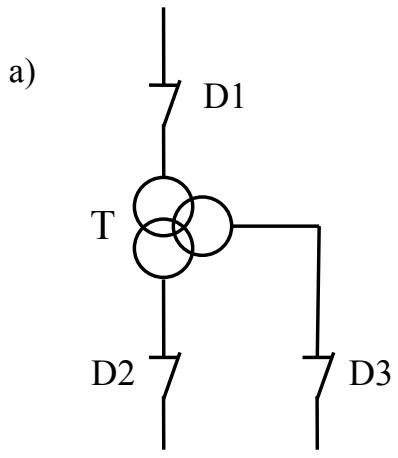

b)

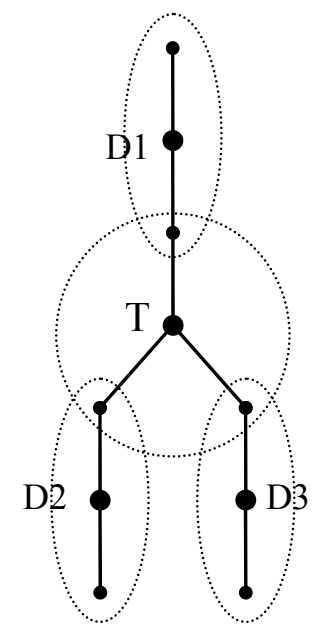

Figure 2. Power three-pole transformer (T) with the adjacent disconnectors (D1, D2 и D3): a - the pictorial image, $\mathrm{b}$ - the corresponding fragment of the stellar graph

In the stellar model each device of the electrical network is represented by the star - the undirected starred graph. The central vertex of the star represents the device itself; and the peripheral vertices of the star represent the connection nodes of the device with the adjacent devices in the circuit. The star has as many rays as there are connection nodes of the device with the adjacent devices. The central vertex of the device star will be called device node.

Thus, breakers and disconnectors are represented by the two-ray stars (Figure 1). Two-pole transformer - by two-ray star, three-pole - by three-ray one (Figure 2). Power line without taps is represented by the two-ray star. Bus section star has as many rays as this bus section has connections, etc.

The join of stars of all electrical devices of the network will be called a stellar graph of the network. Stellar graph can represent a scheme of one power object - the power plant or substation. Also the stellar graph can represent a set of power objects, interconnected by power lines. Stellar graph of a single power object will be denoted as ObjNet. Stellar graph of the whole considered network will be denoted as simply Net.

Formulas and algorithms presented in the article for the analysis of topological states relate to this stellar model. However, the principles of construction of the formulas are the same for any type of graph models of switching circuits - both for more detailed models and for the less detailed ones. Formulas proposed in the article will vary 
insignificantly in case of a transition to another type of graph model.

Effective tool for the switching circuits analysis is provided by a special system of operations on undirected graphs - algebra of graph connectivity (Golovinskii, 2001; Golovinskii, 2013). The basis of this algebra is formed by five binary operations on undirected graphs. These graphs are considered as subgraphs of some graph-universe $\mathrm{U}$. Let A and B be arbitrary subgraphs of undirected graph-universe $\mathrm{U}$. The basic operations of the algebra of graph connectivity are the following:

$$
\begin{aligned}
& \mathrm{A}+\mathrm{B}-\text { union of graphs } \mathrm{A} \text { and } \mathrm{B} ; \\
& \mathrm{A} \& \mathrm{~B}-\text { intersection of graphs } \mathrm{A} \text { and } \mathrm{B} ; \\
& \mathrm{A}-\mathrm{B}-\text { difference of graphs } \mathrm{A} \text { and } \mathrm{B} ; \\
& \mathrm{A} * \mathrm{~B}-\text { increment of graph } \mathrm{A} \text { in graph } \mathrm{B} ; \\
& \mathrm{A} \wedge \mathrm{B} \text { - closure of graph } \mathrm{A} \text { in graph } \mathrm{B} \text {. }
\end{aligned}
$$

The result of each of these operations is again the subgraph of the graph-universe U. This fact allows us to combine given five operations by means of substitutions and thus to create the formulas of any complexity.

Any subset of the set of vertices of the graph-universe is considered as a graph without edges. The first three operations - union, intersection and difference are standard in graph theory. Operations of increment and closure are introduced to calculate the connectivity relations in graphs.

The definitions of these operations are as follows.

1. Union $A+B$. The set of vertices of the graph $A+B$ is the union of the vertex sets of the graphs $A$ and $B$. The set of edges of the graph $\mathrm{A}+\mathrm{B}$ is the union of the sets of edges of the graphs $\mathrm{A}$ and $\mathrm{B}$.

2. Intersection $\underline{A} \underline{A} \underline{B}$. The set of vertices of the graph $A \& B$ is the intersection of the vertex sets of the graphs $A$ and $B$. The set of edges of the graph of $A \& B$ is the intersection of the sets of edges of the graphs $A$ and $B$.

3. Difference A-B. The set of vertices of the graph A-B is the difference of the vertex sets of the graphs $A$ and $B$. This difference is the set of all the vertices of the graph $A$ that do not belong to the graph $B$. The set of edges of the graph $\mathrm{A}-\mathrm{B}$ is the set of those edges of graph $\mathrm{A}$ that are not incident to any vertex of graph $\mathrm{B}$. When we delete the vertices of graph $\mathrm{B}$ from graph A, we should delete all such edges of graph $\mathrm{A}$, in which at least one end belongs to graph $\mathrm{B}$.

4. Increment $\mathrm{A} * \mathrm{~B}$. Increment $\mathrm{A} * \mathrm{~B}$ of the graph $\mathrm{A}$ in the graph $\mathrm{B}$ is the union of the graph $\mathrm{A}$ with all such edges of the graph $\mathrm{B}$, in which at least one end belongs to A. Each added edge is understood as a graph consisting of this single edge and two vertices - the ends of this edge. The set of vertices of graph $A * B$ is the union of the vertex set of graph A and the set of those vertices of graph B that are connected with graph A via an edge of graph $\mathrm{B}$. The set of the edges of the graph $\mathrm{A} * \mathrm{~B}$ is the union of the set of the edges of the graph $\mathrm{A}$ and the set of all such edges of the graph $\mathrm{B}$, in which at least one end belongs to $\mathrm{A}$.

5. Closure $A^{\wedge} B$. The closure of the graph $A$ in graph $B$ (or by means of a graph $B$ ) is the union of graph $A$ with all the connected components of graph $\mathrm{B}$, having a non-empty intersection with the graph $\mathrm{A}$.

The easiest way of the software implementation of the algebra of graph connectivity is to overload (i.e. to redefine) the symbols of the binary operations "+", "\&", "-", "*" and "^", available in $\mathrm{C}++$ language (Golovinskii, 2001). The interpreter of the algebra of graph connectivity is the basis of the Universal Topological Processor (Tumakov, et al., 2011).

In the switching circuits' analysis we need one more basic operation on the subgraphs of graph-universe. The result of this operation, in contrast to the five introduced operations, may not be the subgraph of the original graph-universe. This is the operation of the contraction of one graph by means of the edges of another graph. It is a variation of the concept of the connected homomorphism of undirected graph (Ore, 1962). We denote this operation by the symbol "/" (Aigner, 1997).

Let $\mathrm{B}$ be the subgraph of the undirected graph A. The contraction of one edge of graph A consists in deleting this edge, while its two ends are made coincident. Operation $\mathrm{A} / \mathrm{B}$ can be represented as a sequence of the contractions of all edges of the graph B. To perform this, we need to choose any spanning tree of the graph B and to perform the contraction of all the edges of this tree. The contractions of the spanning tree edges can be done in any order. The edges of the graph A, which do not belong to the selected spanning tree but connect its vertices, are transformed into loops during contraction. Loop is the edge with the coincident ends. All loops are deleted when they appear. 
Graph A will be called contractible graph and graph B will be called contracting graph. The result of the contraction operation $\mathrm{C}=\mathrm{A} / \mathrm{B}$ is not only the graph $\mathrm{C}$, but also the table of the correspondence of the vertices of the contractible graph A to the vertices of contracted graph C.

\section{Results and Discussion}

3.1 Minimizing the Calculations of Electrical Connectivity of the Power Object Elements with the Sources and the Consumers in the Network

Usually the electrical network, in which we select the power object (electrical power plant or substation) for switching circuits' analysis, is not isolated from the neighboring power systems. This network can be connected via transmission lines with adjacent power systems. In these lines the power can flow in one direction or another. While constructing a model of the power network we cut these boundary power lines. The junction points of the simulated electrical network with the adjacent power systems are the nodes of the boundary lines. We regard these points as nodes representing both sources and consumers. Their set is denoted as BoundarySet.

In the switching circuits' analysis of the electrical network it is necessary to take into account the current state of the switching devices. A simple way to do this is to use the set of all disconnected switches. For the case of the whole network, we denote this set as ComsOff, and for the case of a single analyzed power object - as ObjComsOff. The notations of the sets and graphs in the network model are shown in the Table 2. The notations for the graph model of the power object are given in the Table 3.

Table 2. Notations for the graph model of electrical network

\begin{tabular}{ll}
\hline \multicolumn{1}{c}{ Notation } & \multicolumn{1}{c}{ Concept } \\
\hline Net & Stellar graph of the connections of the network electric devices \\
BoundarySet & The set of the boundary power lines' nodes \\
ComsOff & The set of all the disconnected switching devices in the network \\
LinesSet & The set of the nodes of all the lines in the electrical network \\
ZonesGraph & $\begin{array}{l}\text { The zones' graph (represents separately working parts of the power objects } \\
\text { interconnected via power lines) }\end{array}$ \\
SourcesSet & $\begin{array}{l}\text { The set of the vertices of the graph ZonesGraph that represent zones containing } \\
\text { generators } \\
\text { The set of the vertices of the graph ZonesGraph that represent zones containing } \\
\text { ReceiversSet }\end{array}$ \\
\end{tabular}

The graph Net-ComsOff represents electrical connections between the devices over the whole considered electrical network. These connections depend on the state of switching devices. Graph Net-ComsOff is called the switching graph of the electrical network. The graph ObjNet-ObjComsOff represents the electrical connections between the devices of a single power object. It depends on the state of the switching devices. This graph is called the switching graph of the power object.

The nodes of the power lines (central nodes of starred graphs representing power lines), entering to the substation, are not included in the stellar graph of the power object. We include in this graph only the connection nodes joining power lines with the devices of this power object. These nodes will be called entry points (or entries) of power lines into the power object. We select two subsets of the set of the entries of power lines into the power object. First is the set of the upstream power lines' entries. Second is the set of the downstream power lines' entries. The first set is denoted as SupplyLineEntries, the second - FeedLineEntries. Entry point of upstream line is connected to the voltage sources at the electrical network by the closed chains, not passing through those busbars, to which this power line is connected at the current state of the switches. The same way, the downstream power line entry point is connected to the consumers by closed chains, not passing through those busbars, to which this power line is connected at the current state of the switches. The sets SupplyLineEntries and FeedLineEntries may have non-empty intersection, since the same power line can be both supplying and supplied with respect to this power object. 
Table 3. Notations for the graph model of the power object

\begin{tabular}{|c|c|}
\hline Notation & Concept \\
\hline ObjNet & Stellar graph of the connections of the power object devices \\
\hline VoltSet & The set of the nodes of the power devices under voltage in the power object \\
\hline SupplyLineEntries & The set of the entry points of the upstream power lines \\
\hline FeedLineEntries & The set of the entry points of the downstream power lines \\
\hline EquipSet & $\begin{array}{l}\text { The set of nodes of all power devices in the power object (transformers, bus sections, } \\
\text { power lines' entries, reactors) }\end{array}$ \\
\hline BusSet & The set of the nodes of all bus sections in the power object \\
\hline TransSet & The set of the nodes of all power (auto)transformers in the power object \\
\hline ObjComsOff & The set of the nodes of all disconnected switching devices in the power object \\
\hline
\end{tabular}

Complex SCADA/EMS can obtain additional, but incomplete, information about what power line entries of the power object are connected to the sources, and what are connected to the consumers. This information can be obtained from the ammeters and from the load flow calculations. If at the entry point of the power line the active power flow is directed towards the power object, then we can conclude that the power line is upstream. However, this feature can not determine whether this line simultaneously is downstream. The same way, if the flow of active power at the entry of a power line is directed outwards the power object, the power line is downstream. However, this feature can not determine whether this line simultaneously is upstream.

When performing the switching circuits' analysis for selected power object (power plant or substation) we consider the elements of the set SupplyLineEntries as sources, and the elements of the set FeedLineEntries - as consumers. These sets depend on the current status of the electrical connections in the whole network. They are subject to change for each switching in the network. The calculation of the sets SupplyLineEntries and FeedLineEntries requires the analysis of the electrical network topology beyond the boundaries of selected power object. In general, this calculation requires the analysis of the topology of the entire network. This analysis can require excessive memory and time expenses. However, we are talking about not any task of the topological analysis of the power network, but in fact about a single special task (having two variants - to find connections to sources and to find connections to consumers). This enables significantly simplify the solution. It consists of the following.

As a result of the disconnection of the switching devices the power object can be divided into parts, which are not connected to each other by closed circuits within the power object. These parts will be called the zones of the power object. In particular, the end of the power line disconnected from the substation buses will be the zone of power object. Such a zone like the disconnected end of power line may contain, in particular, closed line disconnector. Moreover, such a zone may contain no other elements, except the pole (from the line side) of the open line disconnector. The zones of the power object are in one-to-one correspondence with the connected components of the switching graph ObjNet-ObjComsOff of this power object.

Let us build the graph that represents the connections of power objects' zones via power lines over the entire electrical network. This graph will be called the zones' graph of the power network. The vertices of this graph will represent the nodes of the power lines and the power objects' zones. This graph we can build by joining the starred graphs of all the power lines, making coincident those peripheral vertices that belong to the same zone. The edges of these starred graphs will connect the zones of different power objects through the nodes of the power lines. This graph of the power objects' zones will have much smaller dimension than the switching graph Net-ComsOff of the network. Nevertheless, the analysis of the zones' graph allows to establish for any entry of the power line, whether this entry is connected to the sources and to the consumers in the network by the closed chains not passing through the power object buses, connected with the this entry via closed chains.

We denote the zones' graph of the network power objects through ZonesGraph. During the switchings at the substations the set of the edges of the graph ZonesGraph will not change. Only the vertices of this graph will change. Hence we find the dimension of the graph ZonesGraph. The number of its edges is $L+2 M$, where $L$ is the number of junction points of the simulated network with the adjacent power systems, $M$ is the number of the internal electrical power lines of the simulated network. The number of the vertices of any connected graph is not greater than the number of its edges plus one. Hence we find that the number of the vertices of the graph ZonesGraph is not greater than $L+2 M+N$, where $N$ is the number of connected components of zones graph ZonesGraph. The analysis of the topology of the graph with this number of the vertices and edges does not meet the dimension problem. 
As a result of the disconnection of the switching devices a zone can be divided into two zones. As a result of closure of the switches two zones may join into one. Therefore, when switching a single switching device, there is no need to re-build the entire graph ZonesGraph. Suffice it to update the subset of its vertices, representing the zones of that power object, where there was a switching, and to adjust the references to these vertices from the incident edges.

At the beginning of the work of the SSM program the graph ZonesGraph needs to be fully calculated. This is accomplished by the contraction of the network switching graph Net-ComsOff through all its edges, except those, which are incident to the nodes of the power lines. The set ComsOff should represent all currently disconnected switches in the network. Let us denote the set of nodes of all internal and boundary power lines in the given electrical network through the LinesSet. Then the contracting graph will be expressed by the formula

$$
\text { Net - ComsOff - LinesSet . }
$$

The zones' graph will be expressed by the formula

$$
\text { ZonesGraph }=\text { (Net-ComsOff) } / \text { (Net-ComsOff-LinesSet) } \text {. }
$$

Figure 3 shows the simplified network containing four substations, designated as SS-1, SS-2, SS-3 and SS-4. Filled squares mean closed breakers, not filled - disconnected breakers. The substation SS-1 is divided into two zones by the disconnected section breakers. Figure 4 shows zones graph ZonesGraph for this network. The vertices SS-1-1 and SS-1-2 represent the zones of substation SS-1.

When performing the contraction operation (1) the table of the correspondence of the vertices of the contractible graph Net-ComsOff to the vertices of the resulting graph ZonesGraph is compiled. Using this table, we find the vertices of the graph ZonesGraph, representing all those connected components of the electrical power stations that contain generators. Let us denote the set of these vertices through SourcesSet. Let us find also the vertices of the graph ZonesGraph, representing all those connected components of the power objects that contain consumers. The set of these vertices is denoted through ReceiversSet.

Let LineEntry be the entry point of any power line entering the analyzed power object. The vertex LineEntry belongs to the switching graph Net-ComsOff. Using the table of correspondence of the vertices of the graph Net-ComsOff to the vertices of the graph ZonesGraph we find the vertex of the latter, which corresponds to the entry point LineEntry. This vertex of the graph ZonesGraph we denote as EntryZone. 


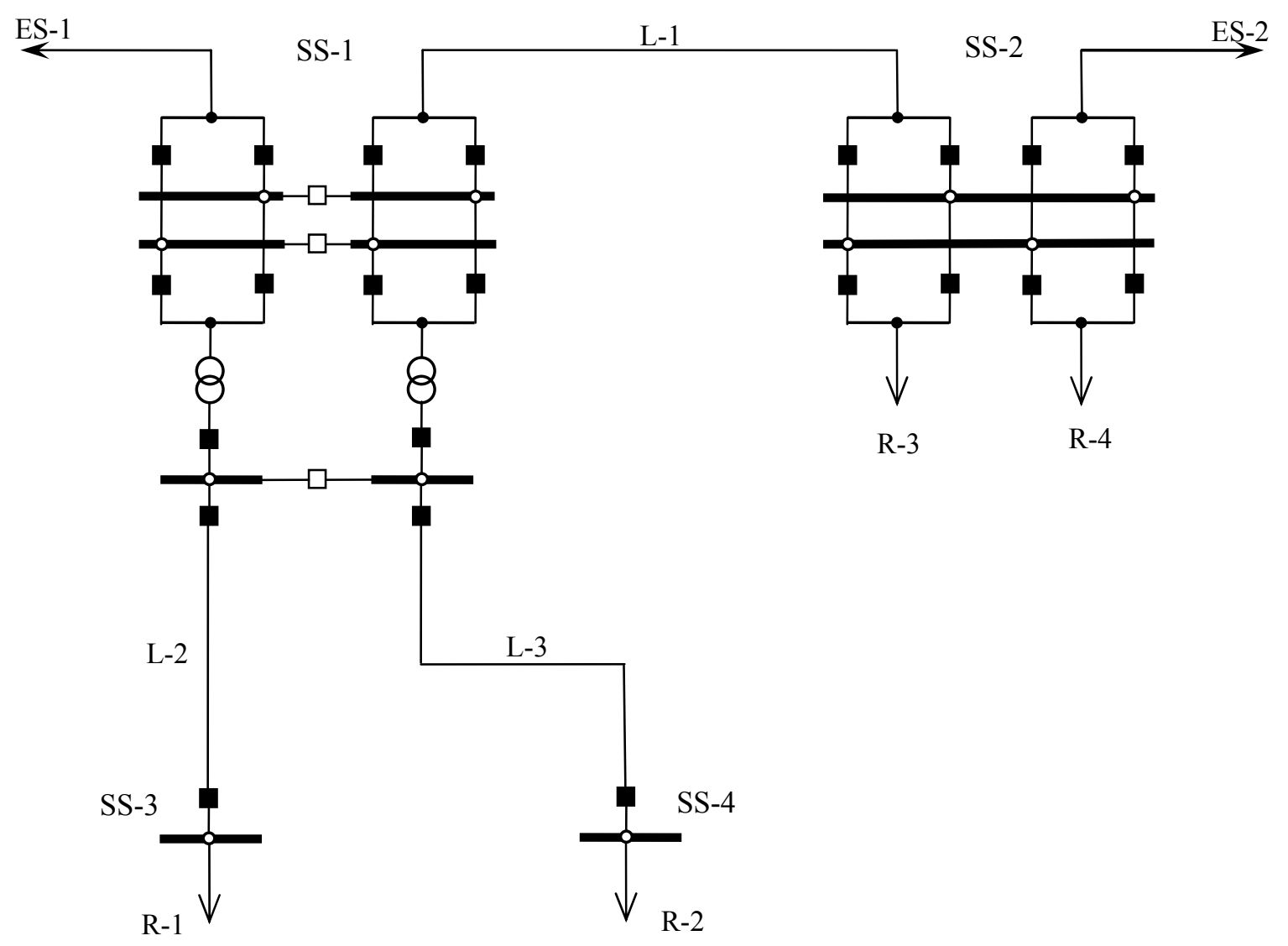

Figure 3. Simplified scheme for the network consisting of four substations

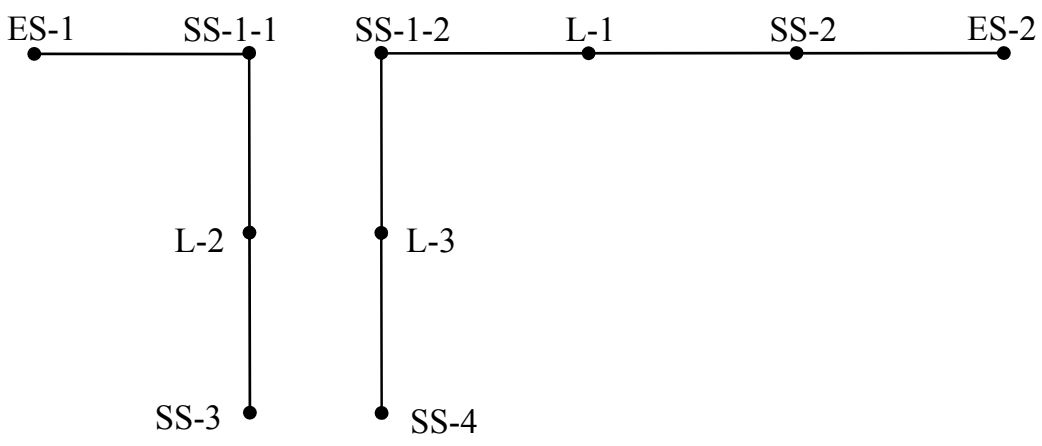

Figure 4. The graph of the power objects' zones of the network, depicted on the figure 3

Let LineNode be the node of the power line, the entry of which to the considered power object is LineEntry. The vertex LineNode can by easily defined by the vertex LineEntry. At the current states of switching devices the entry point LineEntry belongs to the set SupplyLineEntries if and only if the node LineNode is connected via paths in the graph ZonesGraph, bypassing the vertex EntryZone, with at least one vertex of the set SourcesSet in the graph ZonesGraph. The latter case occurs if and only if the following subset of the set of vertices of the graph ZonesGraph is not empty:

$$
\text { (LineNode }{ }^{\wedge} \text { (ZonesGraph - EntryZone)) \& (SourcesSet + BoundarySet). }
$$

A similar formula expresses a criterion for the entry point LineEntry membership of the set FeedLineEntries. 
This membership takes place if and only if the following set is not empty:

$$
\left(\text { LineNode }{ }^{\wedge} \text { (ZonesGraph - EntryZone) }\right) \&(\text { ReceiversSet + BoundarySet }) .
$$

To construct the sets SupplyLineEntries and FeedLineEntries for the power object, you need to look over all the entries of the power lines into given power object and to perform for each entry the described checks for membership of these sets.

In case of a single switching operation in the network, there is no need to re-build the entire graph ZonesGraph by the formula (1). Suffice it to update the set of the vertices of this graph, representing the zones only of that power object, where the switching has been executed. Accordingly, it is necessary to adjust the set of the graph ZonesGraph edges, incident to these vertices. Formally, this operation must be carried out with the same formula (1). The latest, in this case, should be applied to the power network, consisting of a single analyzed power object. The nodes of the power lines entering to this power object will be the interface points of the given a small electric network with external power system. After that, the necessary changes should be entered into the database: into the table of vertices and edges of the graph ZonesGraph and into the table of the correspondence of the vertices of the graph Net-ComsOff to the vertices of the graph ZonesGraph.

Having received the sets SupplyLineEntries and FeedLineEntries accordingly to current switching state of the power network, we may begin to analyze the topological state of this substation. This no longer requires going beyond the boundaries of substation. Now the establishing of the topological characteristics of individual substation devices, it's group electrical installations, and the substation as a whole will be reduced to the calculation of the subgraphs of the graph ObjNet-ObjComsOff by means of the operations of the algebra of graph connectivity.

To begin, we present a formula which gives the set VoltSet of the nodes of the power devices under voltage at the substation:

$$
\text { VoltSet }=(\text { SupplyLineEntries ^ }(\text { ObjNet }- \text { ObjComsOff })) \& \text { EquipSet } .
$$

The meaning of this formula is as follows. The substation switching graph ObjNet-ObjComsOff may consist of a number of connected components. They correspond to zones of the substation. In this set of connected components the closure operation SupplyLineEntries ${ }^{\wedge}\left(\mathrm{ObjNet}^{-} \mathrm{ObjComsOff}\right)$ selects those components that contain the elements of the set SupplyLineEntries - the entries of upstream power lines, i.e., "sources". The connected components, containing no sources, are rejected. Only those device nodes that belong to the connected component containing the "sources" will be under voltage. To get the set of nodes of power devices under voltage, we find the intersection of the graph SupplyLineEntries ${ }^{\wedge}\left(\mathrm{ObjNet}^{-O} \mathrm{ObjComsOff}\right)$ with the set of nodes of all power devices, i.e. EquipSet.

The following sections describe the examples of the calculation of the topological states.

\subsection{Analysis of the Topological State of Individual Devices at the Substation}

Example 1. Suppose we want to know whether a given bus section at the substation is on load. Let us denote as Bus the central vertex of the starred graph, representing this bus section.

The topological state "on load" for the bus section is defined according to the following topological rule: this state takes place if and only if at least one connection node of this bus section is connected within the substation with the entries of the upstream power lines via closed chains, not passing through the node Bus, while another connection node of this bus section is connected within the substation with the entries of the downstream power lines via closed chains, not passing through the node Bus.

This topological criterion can also be expressed by the following definition. The analyzed bus section is on load if and only if the following three conditions are simultaneously fulfilled:

1) at least one connection node of the bus section is connected with the entries of the upstream power lines via the closed chains within the substation, not passing through the node Bus;

2) at least one connection node of the bus section is connected with the entries of the downstream power lines via the closed chains within the substation, not passing through the node Bus;

3 ) the total number of the connection nodes of the bus section, each of which is connected with at least one entry of the power lines via closed chains, not passing through the node Bus within the substation, is greater than 1.

Let us denote as BusPolesSet the set of all the connection nodes of the analyzed bus section. This set is expressed by the formula 


$$
\text { BusPolesSet }=\text { (Bus } * \text { ObjNet })- \text { Bus. }
$$

The meaning of this formula is as follows. Graph Bus*ObjNet is the starred graph representing considered bus section. If you subtract, as a graph, the central vertex Bus from this star, then along with this vertex there will be removed all edges, incident to this vertex, i.e. all the rays of the star. Only the ends of the rays will remain. They form the set of connection nodes of the considered bus section with the adjacent devices.

Let us denote by SPoles the subset of the set BusPolesSet, consisting of those nodes that are connected within the substation via the closed chains, not passing through the node Bus, with the entries into the substation of the upstream power lines. The set SPoles is expressed by the formula

$$
\text { SPoles }=\left(\text { SupplyLineEntries }{ }^{\wedge}(\text { ObjNet }-(\text { ObjComsOff }+ \text { Bus }))\right) \& \text { BusPolesSet } .
$$

This formula is obtained as follows. The graph ObjNet-(ObjComsOff+Bus) is the graph of all closed chains at the substation, not passing through the node Bus of the bus section. The graph SupplyLineEntries ${ }^{\wedge}\left(\mathrm{ObjNet}^{-}(\mathrm{ObjComsOff}+\mathrm{Bus})\right)$ is the union of all those closed chains within the substation that contain the entries of the upstream power lines. The intersection of this graph with the set BusPolesSet gives the set of the connection nodes of the analyzed bus sections that are connected by the closed chains, not passing through the Bus, with the entries of upstream power lines.

The same way we denote by FPoles the subset of the set BusPolesSet, consisting of those nodes that are connected within the substation via the closed chains not passing through the node Bus, with entries to the substation of downstream power lines. The set FPoles is expressed by the formula

$$
\text { FPoles }=\left(\text { FeedLineEntries }{ }^{\wedge}(\text { ObjNet }-(\text { ObjComsOff }+ \text { Bus }))\right) \& \text { BusPolesSet } .
$$

According to the above mentioned topological criterion, the analyzed bus section is on load only when both sets SPoles and FPoles are not empty, and their union SPoles+FPoles consists of at least two elements.

The same way the presence of the "on load" status is checked for any other devices at the substation. The other topological states for different types of devices at the substation are determined by the use of algorithms of the same nature.

\subsection{Analysis of the Topological State of the Group Electrical Installations}

Let us consider the algorithms recognizing topological states for the group electrical installations: a switchgear, a pair of parallel transformers, a join of the power line with the switchgear, power lines group of possible transit through the substation.

Example 2. Let us find the set of switchgear bus sections, operating jointly with this bus section. Let TransSet be the set of nodes of power transformers at the substation. Each connected component of the graph ObjNet-TransSet represents one of the switchgears of the substation. Each connected component of the graph ObjNet-TransSet-ObjComsOff, or ObjNet-(ObjComsOff+TransSet), represents one of the electrically connected parts of the switchgear. Let Bus be any bus section node at the substation. Graph Bus $^{\wedge}($ ObjNet-(ObjComsOff+TransSet) $)$ represents the connected part of the switchgear that contains the bus section Bus. The set of all the bus sections, connected with Bus by the closed chains within the switchgear, is expressed by the formula

$$
\left(\text { Bus }^{\wedge}(\text { ObjNet }-(\text { ObjComsOff }+ \text { TransSet }))\right) \& \text { BusSet . }
$$

This formula gives the required set of the switchgear bus sections, operating jointly with the bus section Bus.

Example 3. Let us consider the recognition of the topological state of the pair of the parallel transformers. Let the substation contain two similar two-pole transformers, connected in the same way with the bus systems and other devices. Let us calculate, whether there is electrical connection between these transformers within the switchgears at their higher (HV) and low (LV) voltage levels.

Let Trans1 and Trans2 be the central vertices of starred graphs of these transformers. Let Pole1 be the connecting node of the first transformer at its HV; Pole2 be the corresponding connecting node of the second transformer. Graph

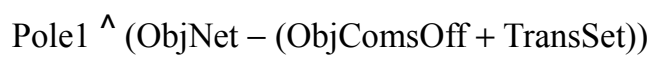

is the union of all the closed chains at a substation, containing the node Pole 1 and not passing through the transformers. Two transformers are connected via a closed chain within the switchgear at their HV if and only if this graph contains the node Pole2. 
By the same formula (2) the presence of the closed chain between the transformers within the switchgear at their LV is checked. In this case Pole1 and Pole2 will represent the connecting nodes of the transformers at their LV side.

Having established the presence or absence of connections between the transformers via closed chains within the switchgears at the $\mathrm{HV}$ and $\mathrm{LV}$, we find the topological state of the given group electrical installation in accordance with the definition of topological states shown in the Table 1.

The topological states of a pair of parallel three-pole transformers are calculated in the same way. In this case the formula (2) should be applied also to the switchgear of a middle voltage level since this switchgear can contain closed chains connecting considered transformers.

Example 4. A power line entering the substation may be connected via closed chains within the switchgear with not every bus section of the switchgear, to which it is attached. Suppose for a given power line it is required to find that part of the switchgear (the set of jointly operating bus sections), to which this power line is connected via closed chains at the current state of the switches.

Let LineEntry be the entry node of this power line to the substation. Then the set of the nodes of all required bus sections will be expressed by the formula

$$
\left(\text { LineEntry }{ }^{\wedge}(\mathrm{ObjNet}-(\mathrm{ObjComsOff}+\text { TransSet }))\right) \& \text { BusSet . }
$$

The group electrical installation understood as the power line join with the switchgear is in the switching state "the connection is present" if and only if this set of bus sections is not empty.

Example 5. For a given power line we will find all the power lines, with which the first power line carries out the electricity transit through the substation. Let LineEntry be the entry node of the given power line into the substation. The set of nodes of all the entries of power lines to the substation we denote as LineEntriesSet. Obviously, LineEntriesSet $=$ SupplyLineEntries + FeedLineEntries .

The set of the entry nodes of the power lines, connected to the entry LineEntry via the closed chains within the switchgear, will be expressed by the formula

$$
\left(\text { LineEntry }{ }^{\wedge}(\text { ObjNet }-(\text { ObjComsOff }+ \text { TransSet }))\right) \& \text { LineEntriesSet . }
$$

This set contains the entry LineEntry. It seems not correct to assume that the power line carries out transit to itself. If so, it is necessary to subtract the entry LineEntry from the found set. If the initial power line has a parallel power line falling into this set, then this parallel line should be deleted as well.

\subsection{Analysis of the Topological State of the Substation as a Whole}

Example 6. One of the topological characteristics of the substation as a whole is a description of its zones separately operating parts. The zone is of interest primarily if it contains bus sections that are in a state "on load". The equivalent of this criterion is that the "operating" zone must contain at least one entry of the upstream power line and at least one entry of the downstream power line, while the total number of power lines entries into this zone should be not less than two.

Example 7. Let us consider the problem of the division of the substation accordingly the voltage levels. The area of identical voltage class equipment is switchgear. The set of such areas of the substation is in one-to-one correspondence with the set of connected components of graph ObjNet-TransSet. Such a connected component will be called the graph of the switchgear. Most often, the substation contains no more than one switchgear of the given voltage class. In the circuits of the auxiliary power of the substation several switchgears of the same voltage class are possible.

The switchgear operates separately from the other parts of the substation exactly when it consists of one or more zones not exceeding its boundaries. This means that each connected component of the switching graph ObjNet-ObjComsOff is either the subgraph of the graph of the switchgear or it does not intersect with this graph This criterion can be succinctly expressed by the operation of the graphs' closure. For this we denote as SwitchGear the graph ObjNet-TransSet connected component, representing considered switchgear. Then the specified criterion will be expressed by the equality condition

$$
\text { SwitchGear }^{\wedge} \text { (ObjNet-ObjComsOff) }=\text { SwitchGear. }
$$

Example 8. Let us consider the topological characteristic expressing the violation of the transit through substation at its highest voltage level. This state occurs when both of the following conditions are fulfilled:

1) the switchgear of the highest voltage level is in separate operation (is divided); 
2) the substation as a whole is not divided.

Checking the first condition is described in Example 2. The second condition is satisfied if and only if the graph ObjNet-ObjComsOff has a connected component containing two separately operating parts of the switchgear of highest voltage level.

Considered topological characteristics of the substation equipment may be subject to the persistent dispatch monitoring. This information is important in itself for dispatcher. At the same time, many other topological characteristics of the equipment state not discussed in the paper are necessary to control the permissibility of switching operations.

The technical regulations for executing the operative switchings in electrical networks contain requirements, having the character of logical locks for the switching operations (GOST 2013; Instruction for switchings in electrical facilities, 2004). According to them, permission or blocking switching of the switching device is defined by the topological state conditions on the equipment associated with this device.

\section{Conclusion}

The tasks of the control of the topological state of the electrical substations circuits constitute an important part of operational-dispatch control of the electrical network mode. The article highlights the classes of these problems: the control of the topological state of the individual power devices, group electrical installations, substation as a whole. The mathematical methods for the automatic calculation of the topological states have been offered. The basis of these methods is a special system of operations with graphs. It belongs to the field of the topological methods for the analysis of the contact circuits. In contrast to the algorithms, using only Boolean algebra, the topological algorithms do not require processing when transferring control programs from one substation to another, which has a different topology. The offered algorithms are simply and succinctly expressed by the formulas of the algebra of the graphs, which greatly simplifies their implementation.

One of the directions of further developments should be the application of the offered mathematical formalism of the graph algebra to the topology of power networks and substations diagrams, specified in the CIM format. The topological structure of CIM is a graph, the vertices of which represent the CIM objects and the edges represent the relations between the objects by the hierarchy and by association (with the help of cross-references). The application of graph algebra to the analysis of the CIM topology will further enhance the generality and reliability of the control programs of the topological state of the substations.

\section{Acknowledgments}

The paper presents the results of the work done within the applied research topic: "Development of the design principles and main technical solutions of a new generation $110 \mathrm{kV}$ digital substation of a high degree of prefabrication" (the unique identifier of the applied research work and experimental development RFMEFI57914X0033) in Zelenograd innovation and technology centre. The work is financially supported by the Ministry of education and science of the Russian Federation.

\section{References}

Aigner, M. (1997). Combinatorial Theory. Berlin: Springer. http://dx.doi.org/10.1007/978-3-642-59101-3

Aranovich, B. (1947). Matrix methods for analysis and synthesis of relay-contact circuits. Ph.D. thesis in engineering science.

Aranovich, B. (1949). The use of the matrix methods in problems of the structure analysis of the relay-contact circuits. Automation and Remote Control, 10(6), 437-451.

Gikinskaya, A., \& Lyubarskii, Y. (2003). The automatic analysis of the topology of the network electrical circuits in the automated systems for dispatch control at the power interconnection utilities. Elektricheskie Stantsii (Electrical Power Stations), 11, 22-26.

Gikinskaya, A., Lyubarskii, Yu., \& Chuvakov, A. (2007). The automation of the dispatch analysis of the situations at the energy system in a shortage of operational information. Electrichestvo(Electricity), 5, 12-17.

Golovinskii, I. (2001). Object-oriented approach to the development of the programs for switching circuits' analysis of the electrical networks. Izvestiya RAN. Energetika (Transactions of the Russian Academy of Sciences. Energetics), 2, 46-56.

Golovinskii, I. (2013). The development of the methods and algorithms for the automation of planning and control of the operative switchings in the electrical networks of power systems. Author's abstract of the D. 
Sc. of engineering. Moscow: JSC "RDC FGC UES". Retrieved November 10, 2014, from http://www.ntc-power.ru/upload/medialibrary/fac/Avtoreferat_Golovinsky.pdf.

GOST P 55608-2013. United power system and isolated power systems. Operative-dispatch management. Switching in electrical facilities. General requirements. (2014, July 1).

IEC 61968-11. Application integration at electric utilities - System interfaces for distribution management Part 11: Common information model (CIM) extensions for distribution. (n.d.).

IEC 61970-301. Energy management system application program interface (EMS-API) - Part 301: Common information model (CIM) base. (n.d.).

Instruction for switchings in electrical facilities. CO 153-34.20.505-2003. (2004). Moscow: The publishing house SC ENAS.

Kupershmidt, Yu., \& Lyubarskii, Yu. (1985). The question programming for the building of automated information systems. Teleinformational systems of real time for the dispatch energy systems' control. The collection of the scientific works of VNIIE. Moscow: Energoatomizdat.

Lunts, A. (1950a). The application of the Boolean matrix algebra to the analysis and synthesis of the relay-contact circuits. Doklady AN SSSR (Proceedings of the USSR Academy of Sciences), 70(3), 421-423.

Lunts, A. (1950b). The synthesis and analysis of the relay-contact circuits with the help of characteristic functions. Doklady AN SSSR (Proceedings of the USSR Academy of Sciences), 75(2), 201-204.

Lunts, A. (1952). The algebraic methods for the analysis and synthesis of the contact circuits. Izvestiya AN SSSR. Seriya Matematicheskaya (Izvestiya: Mathematics), 16, 405-426.

Lyubarskii, Yu. (1978). The automation of the analysis of situation in the dispatch information systems. Elektricheskie Stantsii (Electrical Power Stations), 11, 13-17.

Lyubarskii, Yu. (2003). The automatic control of the work state of the equipment for the dispatch control. Vestnik VNIIE-2003 (Bulletin of VNIIE-2003), 130-135.

Ore, O. (1962). Theory of Graphs. American Mathematical Society.

Stanković, R., Astola, J., \& Karpovsky, M. (n.d.). Some historical remarks on switching theory. Retrieved November 10, 2014, from http://mark.bu.edu/papers/200.pdf.pdf

Tumakov, A., Golovinskii, I., Londer, M., \& Diachenko, M. (2011). Universal topological processor for the systems of electrical networks' intelligent control (UNITOP). State Registration certificate for the IBM program \# 2011613357. Registered in 29.04.2011. The right holder: JSC "Decima" (RU).

Uslar, M., Specht, M., Rohjans S., Trefke, J., \& Gonzalez, J. M. (2012). The Common Information Model CIM. Berlin Heidelberg: Springer-Verlag. http://dx.doi.org/10.1007/978-3-642-25215-0

\section{Notes}

Note 1. Currently - Saint Petersburg State Electrotechnical Institute "LETI" named after. V.I. Ulianov (Lenin).

Note 2. The term "stellar model" was taken from S.V. Amelin (the "Modus" company, Moscow).

\section{Copyrights}

Copyright for this article is retained by the author(s), with first publication rights granted to the journal.

This is an open-access article distributed under the terms and conditions of the Creative Commons Attribution license (http://creativecommons.org/licenses/by/3.0/). 